Artículo Original

\title{
Impacto del programa Mindfulness Basado en la Terapia Cognitiva: estudio de caso en la Universidad de Alcalá
}

\author{
Dr. Juan-Carlos Luis-Pascual \\ Vicedecano de la Facultad de Medicina y Ciencias de la Salud, Universidad de Alcalá \\ Autor correspondencia: juan.luis@uah.es
}

Recibido: 15/06/2018; Aceptado: 23/07/2018; Publicado: 30/01/2019

Resumen: En la sociedad actual, el aumento del estrés provocado por una alta exigencia del rendimiento personal y profesional de manera continuada, provoca la necesidad de tener espacios de quietud y serenidad que atenúen o que hagan más llevadero dicho ritmo de vida. El aumento de la consciencia hace más evidente todo el tiempo que una persona pasa realizando las tareas cotidianas de una manera automática. En este contexto surgen diferentes ofertas de propuestas y protocolos que pretenden mejorar la concentración, la conciencia y el estado de bienestar. El objetivo de este estudio es medir la efectividad de un programa de práctica estandarizado de Mindfulness basado en la terapia cognitiva (MBCT) de ocho semanas, llevado a cabo en la Universidad de Alcalá, a través del cuestionario de atención plena de cinco facetas (FFMQ): observación, descripción, actuación consciente, no enjuiciamiento y no reactividad. Todas estas variables mejoran y se mantienen constantes sus efectos a los tres meses, a excepción de la observación y la actuación consciente que remiten ligeramente.

Palabras Clave: Atención Plena; Estrés; Ansiedad; Dolor; REBAP; MBCT; FFMQ; Bienestar; Depresión.

\begin{abstract}
In today's society, the increase in stress caused by a high demand for personal and professional performance in a continuous manner, requires the need to have spaces of calm and serenity that attenuate or make more bearable this rhythm of life. In this context different offers of proposals and protocols that aim to improve concentration, awareness and welfare state arise. The increase in consciousness makes it all the more evident that a person goes through the daily tasks in an automatic way. The goal of this research is testing the effectiveness of a standardized practice program of Mindfulness based on Cognitive Therapy (MBCT) of eight weeks, carried out at the University of Alcalá, is assessed through the five-faceted mindfulness questionnaire (FFMQ): observation, description, conscious action, no prosecution and no reactivity. All these variables improve and their effects remain constant after three months, with the exception of observation and conscious action that remit slightly.
\end{abstract}

Key words: Mindfulness; Stress; Anxiety; Pain; MBSR; MBCT; FFMQ; Well-being; Depression.

\section{Introducción}

Hoy en día nos movemos en una realidad cambiante, que genera un grado de exigencia y activación permanente, tanto a nivel personal como profesional, que pueden llegar a causar episodios de estrés o de ansiedad. En dicho contexto, surgen diferentes ofertas de propuestas y protocolos, que pretenden mejorar la percepción, la actitud y el estado de bienestar. Todo ello supone un cambio de la perspectiva vital, una deconstrucción de la estructura relacional hacia las personas, objetos y 
situaciones, o la necesidad de incorporar espacios de tranquilidad, quietud y serenidad que hagan más llevadero dicho ritmo de vida.

Una de estas intervenciones complementarias está basada en el entrenamiento de la atención plena, que permite afinar y hacer más conscientes las sensaciones corporales (self experiencial), atenuando la hegemonía del discurso mental y su rumiación asociada (self narrativo). La atención plena (o mindfulness) es definido por su creador como "la conciencia que surge al prestar una atención deliberada, en el momento presente y sin juzgar" [1] (p.23). Aunque vamos a encontrar diferentes conceptualizaciones y variadas propuestas de mindfulness que trascienden la mera práctica meditativa, por ejemplo: un estado vital, una actitud, una experiencia que tiene efectos terapéuticos, un modo ser (frente a un modo hacer) o una capacidad propia del ser humano.

El protocolo originario del Dr. Kabat-Zinn para afrontar el estrés a través del mindfulness atiende a las siglas (MBSR) en inglés, o la reducción del estrés basado en la atención plena (REBAP) en español. Dicho programa plantea un compromiso de práctica formal diaria de entre 30' y 45' además de la asistencia durante 8 semanas, de un día a la semana, a un grupo de práctica guiada de 150'. Algunos autores [2] defienden el mantenimiento de un sistema protocolizado y una determinada técnica de enseñanza-aprendizaje para asegurar los efectos del entrenamiento deseados en atención plena en diferentes contextos, con adaptaciones en función del objetivo pretendido: terapia, [3], bienestar [4], educación [5], empresa [6] y deporte [7]. A partir de dicha propuesta inicial han surgido otros programas de intervención basados en mindfulness (MBI), entre ellos, el programa de mindfulness basado en la terapia cognitiva (MBCT) [8].

Podemos destacar la efectividad del mindfulness en la mejora de la autorregulación [9] o en la mejora del bienestar psicológico [10], [11], [12]. Además, dichos efectos podrían perdurar hasta quince meses después de finalizado el curso [13].

Tabla I Intervención pedagógica del mindfulness, basado en [2] (p.107).

\begin{tabular}{|c|c|c|}
\hline Mecanismos psicológicos & Prácticas & Áreas cerebrales \\
\hline 1.-Regulación de la atención. & Meditación de la respiración. & Córtex cingulado anterior. \\
\hline 2.-Conciencia corporal. & $\begin{array}{l}\text { Escáner corporal de las } \\
\text { sensaciones. }\end{array}$ & $\begin{array}{l}\text { Ínsula, junta temporo- } \\
\text { parietal. }\end{array}$ \\
\hline $\begin{array}{l}\text { 3a.-Regulación emocional: } \\
\text { Revalorización. }\end{array}$ & Meditación de las emociones. & Córtex pre-frontal. \\
\hline $\begin{array}{l}\text { 3b.-Regulación emocional: } \\
\text { Exposición, extinción y } \\
\text { reconsolidación. }\end{array}$ & $\begin{array}{l}\text { Meditación de las emociones } \\
\text { difíciles. }\end{array}$ & Amígdala. Hipocampo. \\
\hline $\begin{array}{l}\text { 4.-Cambios en la perspectiva } \\
\text { del self. }\end{array}$ & $\begin{array}{l}\text { Meditación de los } \\
\text { pensamientos. }\end{array}$ & $\begin{array}{l}\text { Cortex cingulado posterior. } \\
\text { Ínsula. }\end{array}$ \\
\hline
\end{tabular}

Hay diferentes variables intervinientes que pueden ocasionar dicha percepción de mejora en los participantes del protocolo de intervención mindfulness: el contexto, el programa de entrenamiento en atención plena, la motivación de los participantes, el instructor-formador, la tipología, las creencias previas y la cohesión del grupo. Entre ellos podríamos destacar que la Universidad de Bangor está a la cabeza del desarrollo y formación de instructores, basados en MBCT y liderados por la Dra. Crane, que han generado unas competencias para la enseñanza de la atención plena, a través de unos criterios de evaluación en la didáctica de la intervención basada en mindfulness (MBI-TAC) que 
permiten profundizar en diferentes aspectos del proceso de enseñanza-aprendizaje, como son: la organización del programa, la gestión del grupo o la relación interpersonal del instructor [14].

Para orientar las propuestas experienciales, se identifican y señalan las correspondencias entre los mecanismos psicológicos, las prácticas y las áreas cerebrales asociadas (ver tabla I).

Entre las fórmulas y métodos que nos podemos encontrar para entrenar la atención plena, unos son formales, asociados a técnicas de meditación y otros informales, relacionados con el hecho de ser más conscientes de las actividades cotidianas. Algunas de las prácticas formales que se detallan, [15] (p.93): -La uva pasa, -Mindfulness en la respiración, -Escáner corporal (Body scan); -Mindfulness caminando, - Mindfulness en los movimientos corporales, -Práctica de los tres minutos. Mientras que se indican [15] (p.118) como principales aspectos de la práctica informal: -Afrontamiento general del día: dividirlo y especificar la intención, -Prácticas formales para la vida diaria,-Prácticas informales específicas para la vida diaria, -Puntos de anclaje, -Recordatorios, -Identificación y uso de las actividades rutinarias y los tiempos muertos, -Diarios de práctica informal,-Mindfulness durante las comidas, -El periodo del sueño.

El mindfulness es una capacidad innata y variable en la persona, [16] que se puede entrenar y medir subjetivamente. Dicha valoración, no es sencilla al ser una estructura compleja que engloba múltiples factores, que deben ser estudiados simultáneamente.

La literatura hallada sobre su medición refuerza la importancia que tiene la evaluación del entrenamiento en la atención plena sobre la experiencia de la práctica. Para ello encontramos diferentes instrumentos: -FFMQ (Five Facet Mindfulness Questionnaire). Cuestionario multifactorial de 39 ítemes que valora las cinco facetas mindfulness [17], [18], [19], [20], [21]-MAAS (Mindful Attention Awareness Scale). Cuestionario unifactorial de 15 ítemes que se centra en la atención/consciencia del mindfulness. Es un instrumento sencillo y se cumplimenta con rapidez, cuya versión española está validada [22] -FMI (Frieburg Mindfulness Inventory). Cuestionario multifactorial de 30 ítemes. [23], [24]. -KIMS (Kentucky Inventory of Mindfulness Skills). Cuestionario multifactorial de 39 ítemes. [17]. CAMS-R (Cognitive and Affective Mindfulness Scale). Cuestionario multifactorial de 12 ítemes en su versión revisada [25]. -MQ (The Mindfulness Questionnaire). Cuestionario unifactorial de 16 ítemes [26]. -PHLMS (Philadelphia Mindfulness Scale). Cuestionario multifactorial de 20 ítemes [27]. -TMS (Toronto Mindfulness Scale). Cuestionario multifactorial de 13 ítemes [28].

El cuestionario FFMQ es uno de los instrumentos más completos para medir la capacidad de atención plena [20], ya que parte de un análisis de las que son las escalas más destacadas, desglosando las características fundamentales de la verdadera esencia del mindfulness. Dicho cuestionario valora cinco facetas: la observación (ser conscientes de todo lo que le rodea), la descripción (ser capaz de explicar cómo uno se siente en un momento dado), la actuación consciente (estando presente y prestando atención a lo que se está haciendo), el enjuiciamiento de la experiencia interior (ser consciente de cada una de las emociones sin evaluar ni experimentar culpa por sentirse de una determinada manera), la reactividad a la experiencia interior (ser consciente de cada una de las emociones sin sentir la necesidad de reaccionar ante ellas).

El cuestionario FFMQ-E ha sido encontrado efectivo para medir la mejora significativa en dichos factores mindfulness para programas MBCT y MBSR con una alfa de Cronbach (que mide la validez del cuestionario) muy elevada [11]. Además, con la escala FFMQ podemos discriminar los meditadores y los no meditadores [20] (p.99) con la valoración global del puntaje obtenido. Resaltando su eficacia en la valoración del impacto del MBCT [19].

\section{Material y Métodos}

Se detallan las características socio-demográficas de los participantes, el procedimiento general para la recogida de los datos, además de las peculiaridades del instrumento que hemos utilizado para este estudio piloto. 


\subsection{Participantes}

La intervención se ha llevado a cabo con un grupo de población no clínica, que participaron libremente en un programa de entrenamiento grupal en atención plena durante ocho semanas. Además, a los participantes de manera adicional se les asignó ejercicios complementarios diarios (prácticas formales), que se llevaban a cabo de forma personal en su casa para completar el entrenamiento grupal semanal, con acceso a lecturas y audios en el Aula virtual de la UAH dispuesta a tal efecto. De los 12 participantes (10 mujeres y 2 hombres) que empezaron el curso, terminaron 11 . Encontrándose la media de edad (ver figura 1) en una horquilla que comprende desde los 41 años hasta los 50.

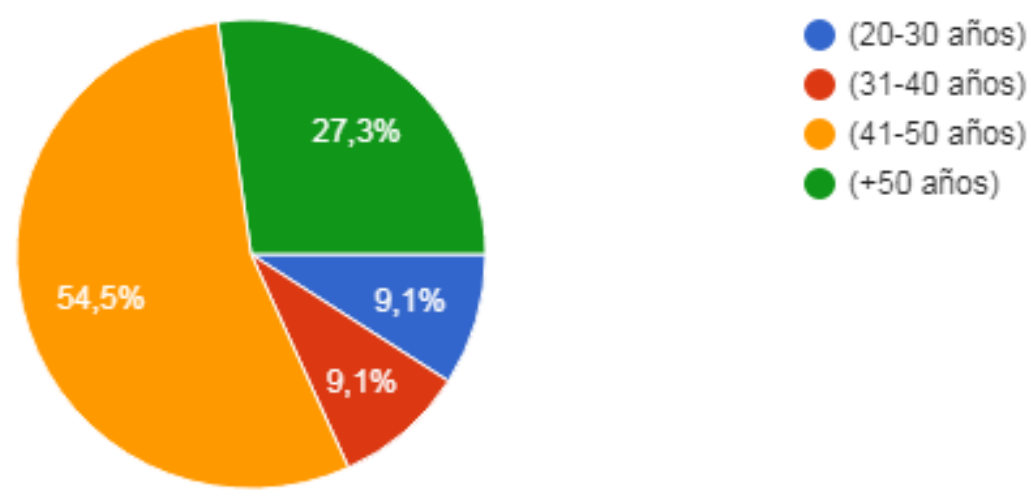

Figura 1 Bandas de edad de la muestra.

Los participantes han cumplimentado voluntariamente un cuestionario FFMQ-E para un pretest, un pos-test, al terminar el curso, y pos-test de seguimiento, a los 3 meses de la finalización de la intervención.

\subsection{Procedimiento}

Este estudio piloto sigue un diseño no aleatorizado descriptivo correlacional de grupo único, con una valoración pre-test, pos-test (finalización) y pos-test (seguimiento tres meses después de la finalización) para determinar las cinco facetas mindfulness que mide la escala FFMQ: capacidad de observar, describir, actuación consciente, enjuiciamiento y reactividad.

Diseño de la intervención: Se lleva a cabo una actividad formativa grupal de ocho sesiones, realizadas periódicamente un día a la semana con una duración de 2 horas y 30 minutos. Las sesiones integran dinámicas, prácticas y ejercicios de entrenamiento de respiración, relajación y atención plena. Además, incluye momentos para compartir y diálogos en grupo sobre las experiencias y dificultades para realizar las prácticas formales e informales, que se trabajaban y practicaban durante las sesiones, con la finalidad de aplicarlos de una forma sistemática en la vida cotidiana.

El objetivo de la investigación consistía en comprobar el impacto y la duración de los efectos del mindfulness de un curso estándar con un Programa MBCT de ocho semanas.

El cuestionario anónimo ha sido enviado a través de una aplicación electrónica invitando a los participantes del curso a tomar parte en dicha valoración de forma voluntaria y sin incentivo económico. Los participantes han tenido opción de no responder a las preguntas entregadas, a excepción de las que formaban parte de la ficha de encuadre. El cuestionario previo fue recogido antes de comenzar el curso MBCT. No ha sido necesario que el investigador enviara recordatorios a los participantes para completar el cuestionario. 
El pos-test fue enviado un día después de terminar el curso y recolectado durante una semana. Los participantes que realizaron el cuestionario después fueron excluidos.

El Programa MBCT tiene una duración de ocho semanas, con una intervención grupal de un día a la semana, de dos horas y media cada día, realizando prácticas de: escáner corporal, movimiento consciente, meditación sedente y el compromiso de una práctica formal en casa de 30-45' diarios para todos los participantes del curso.

El instructor del Programa MBCT está acreditado por la Universidad de Bangor y no interviene en el estudio llevado a cabo.

Los contenidos que se desarrollaron en las sesiones fueron los siguientes (ver tabla II).

Tabla II Secuenciación de los contenidos del curso del Programa MBCT.

\begin{tabular}{cc}
\hline Sesiones & Contenidos \\
\hline Primera & Conciencia y piloto automático \\
\hline Segunda & Viviendo en nuestras mentes \\
\hline Tercera & Centrar la mente dispersa \\
\hline Cuarta & Reconocer la aversión \\
\hline Quinta & Permitir y aceptar \\
\hline Sexta & Los pensamientos no son hechos \\
\hline Séptima & Retiro intensivo \\
\hline Octava & Cómo puedo cuidar de mí mismo \\
\hline Novena & El futuro: mantener y extender lo aprendido \\
\hline
\end{tabular}

\subsection{Instrumento}

Utilizamos el cuestionario FFMQ [18] validado al español [29] que fue complementado por 12 participantes en el pre-test, 7 en el pos-test al finalizar el curso y 6 en el seguimiento, a los tres meses de la finalización del entrenamiento grupal guiado. Los cuestionarios incorporan una ficha de encuadre con la edad y el género.

Empleamos el paquete estadístico SPSS (versión 25) para el análisis de los datos y el programa Excel para la elaboración de los gráficos de las figuras aportadas en los resultados. Se hace una revisión preliminar de los datos introducidos para detectar errores antes de llevar a cabo el análisis estadístico descriptivo para hallar las medias, las desviaciones típicas y las diferencias entre los diferentes cuestionarios cumplimentados.

\section{Resultados}

En los datos generales obtenidos del vaciado del cuestionario FFMQ-E se aprecia una mejora de la puntuación general extraída, por una parte del pre-test al pos-test, y por otra manteniéndose los efectos obtenidos a los tres meses de la finalización del Programa de intervención MBCT. 


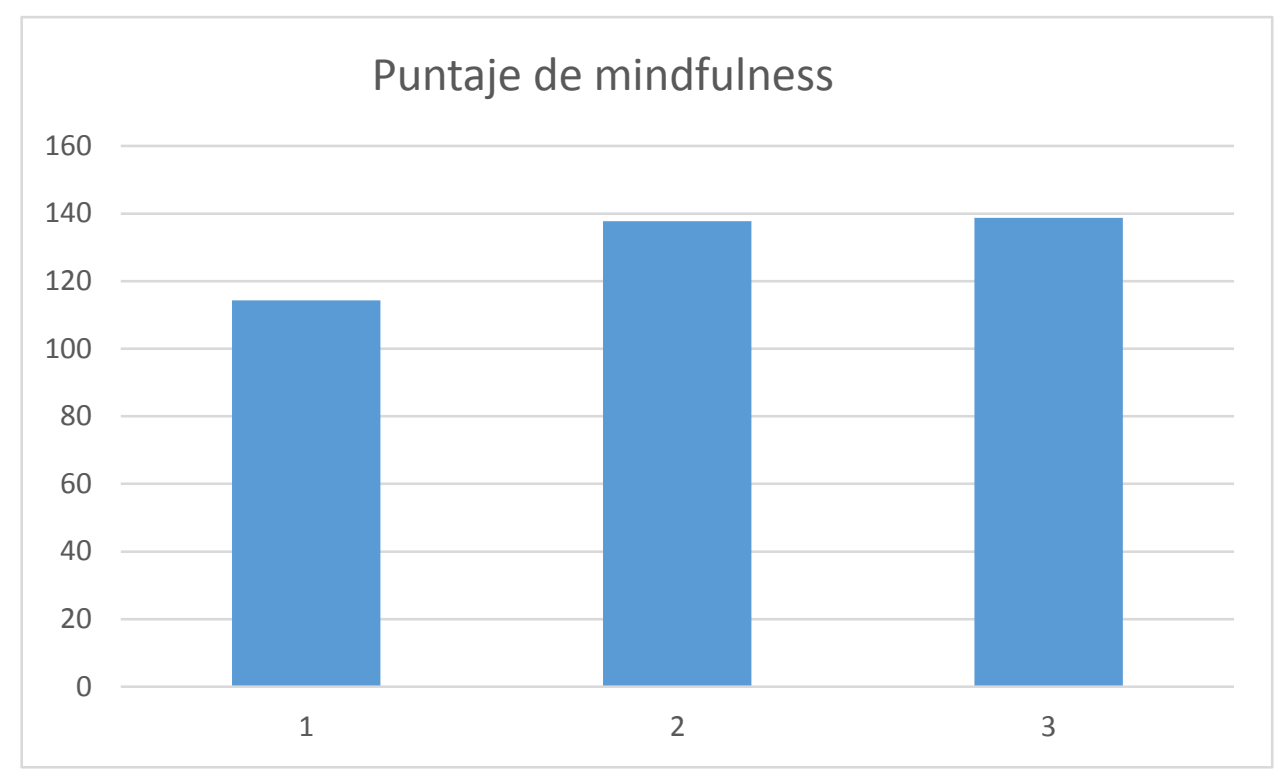

Figura 2 Puntaje global de mindfulness en el pre-post-seguimiento.

En el cuestionario FFMQ-E la puntuación mínima de mindfulness sería 39 y la máxima 195. Podemos observar (ver figura 2) que en el pre-test (1) se obtiene un puntaje de 114, en el pos-test (2) de 137 y en el seguimiento, a los tres meses de acabar (3) de 138.

Tabla III Medias y desviaciones típicas de las facetas mindfulness del FFMQ-E.

\begin{tabular}{|c|c|c|c|}
\hline Facetas mindfulness & FFMQ-pre & FFMQ-post & FFMQ-seguimiento \\
\hline Observación & $X(3,20) \operatorname{SD}(0,83)$ & $X(3,71) \mathrm{SD}(0,80)$ & $X(3,62) \operatorname{SD}(0,16)$ \\
\hline Descripción & $X(3,20) \operatorname{SD}(0,78)$ & $X(3,53) \operatorname{SD}(0,99)$ & $X(3,58) \operatorname{SD}(0,36)$ \\
\hline Actuación consciente & $X(2,77) \mathrm{SD}(0,93)$ & $X(3,43) \operatorname{SD}(0,84)$ & $X(3,35) \operatorname{SD}(0,06)$ \\
\hline No enjuiciar & $X(2,76) \mathrm{SD}(0,79)$ & $X(3,53) \mathrm{SD}(0,68)$ & $X(3,75) \operatorname{SD}(0,16)$ \\
\hline No reaccionar & $X(2,74) \mathrm{SD}(0,85)$ & $X(3,37) \operatorname{SD}(0,74)$ & $X(3,45) \mathrm{SD}(0,13)$ \\
\hline SUMA DE LAS (X) & SUMA X(14,67) & SUMA X $(17,57)$ & SUMA X(17,84) \\
\hline MEDIA DE LAS (SD) & MEDIA SD $(0,83)$ & MEDIA SD $(0,81)$ & MEDIA SD $(0,17)$ \\
\hline
\end{tabular}

Podemos apreciar que dicha tendencia general de mejora se reproduce en cada una de las facetas (ver tabla III) perdiéndose ligeramente los efectos en la observación y atención consciente en el postest (a los tres meses de acabar) y aumentando el puntaje en la descripción, el no enjuiciamiento y la no reacción. 


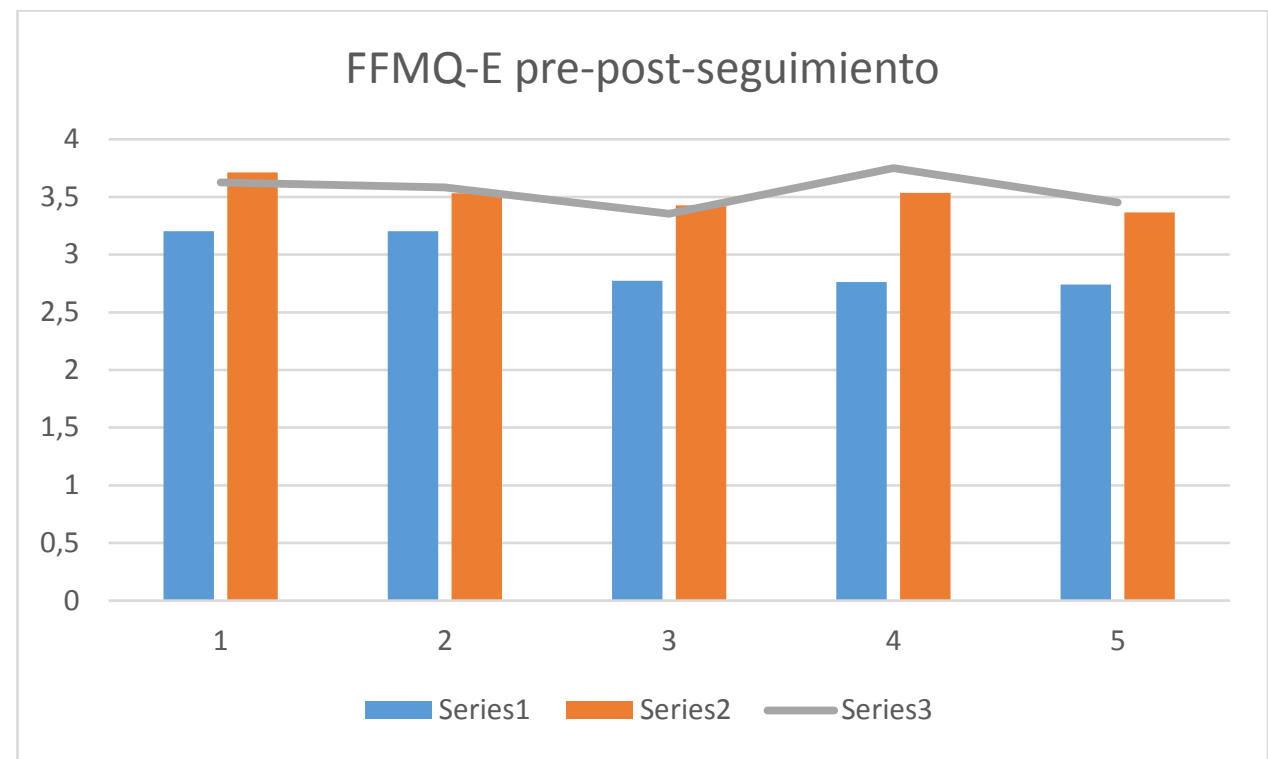

Figura 3 Comparativa de las facetas FFMQ-E en el pre-post-seguimiento.

Observando la comparativa general de las medias (X) de todas las facetas (ver figura 3) se aprecia la mejora de todas ellas en el pos-test, con una horquilla que fluctúa desde $(2,74-3,20)$ en el pre-test (1) hasta $(3,37-3,71)$ en el pos-test (2) con un mantenimiento de los efectos a los tres meses $(3,35-3,75)$ en el seguimiento (3). Siendo la observación (ítem 1), la descripción (ítem 2), la actuación consciente (ítem3) donde se reduce ligeramente en el seguimiento, y donde se aprecia que la mayor mejora absoluta se obtiene en la faceta de no enjuiciamiento (ítem 4), seguida de la de no reactividad (ítem 5), aunque se constata una diferencia en el cuándo. Mientras que la no reactividad (ítem 5) tiene un puntaje mayor relativo en el pos-test, la mayor diferencia se obtiene por el no enjuiciamiento (ítem 4) a los tres meses de finalizar el curso.

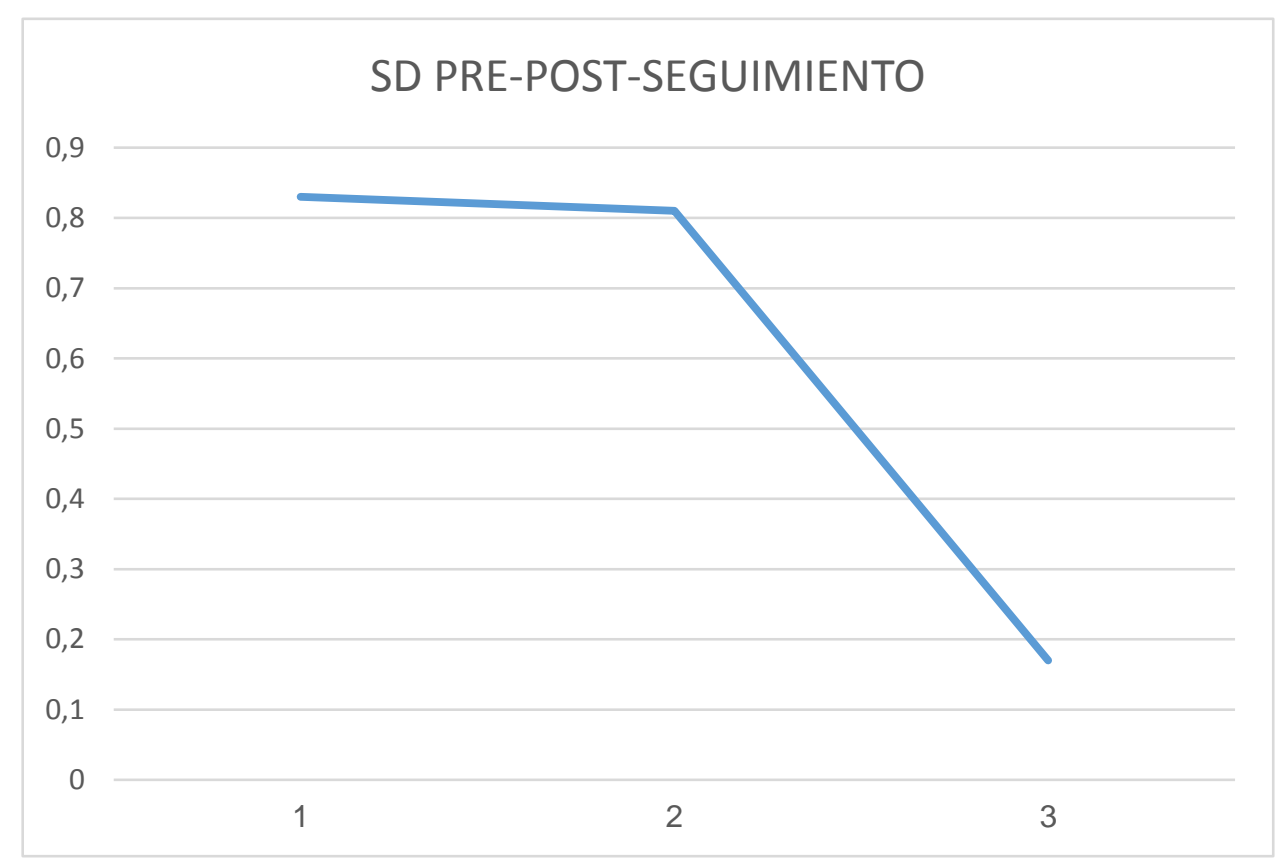

Figura 4 Comparativa de las medias de las SD de las facetas pre-post-seguimiento.

La desviación típica (SD) va siendo cada vez menor (ver figura 4), disminuye desde el pre-test (1) con una SD $(0,83)$, un pos-test $(2)$ con una SD $(0,81)$ hasta el test-seguimiento (3) con una SD $(0,17)$. Se aprecia un gran cambio de tendencia desde el test llevado a cabo al terminar el programa (2) y el test cumplimentado a los tres meses (3). 


\section{Discusión}

Este estudio piloto muestra el impacto positivo generado por un Programa de ocho semanas de MBCT en un grupo de prácticas de atención plena, realizando una sesión semanal de dos horas y media y manteniendo un compromiso de práctica personal en casa.

En relación a la duración de las sesiones presenciales y su separación en el tiempo, tenemos que, si en una MBI se reduce el tiempo de la práctica a dos horas y se espacia el número de sesiones a quince días y no se aprecia una variación significativa en los valores del FFMQ [30]. En esta misma línea, se previene sobre el uso de programas cortos de atención plena con rápidas soluciones [31]. Aunque hay autores que aseveran resultados positivos con sesiones de 20' durante cuatro días [32].

Encontramos estudios equivalentes, como pueden ser el entrenamiento en relajación [33], que también obtienen resultados de impacto positivo en la reducción del estrés [34] en la misma línea del Programa MBCT llevado a cabo.

En relación a los efectos sobre el bienestar psicológico, otras actividades como bailar tango tiene el mismo efecto en la atención plena como la práctica de meditación formal [11], mientras que la práctica de meditación informal se podría desarrollar a través de otras actividades cotidianas como andar, hacer deporte o tocar un instrumento musical.

En el estudio llevado a cabo hay una gran diferencia en el puntaje de todas las facetas del pretest y del pos-test. Se podría afirmar que el Programa MBCT ha tenido un impacto importante en los participantes mejorando las capacidades de observación, descripción, actuación consciente, no enjuiciamiento y no reactividad que se valoran en el cuestionario FFMQ. Las medias de los efectos percibidos por los participantes permanecen prácticamente estables desde la finalización (17,57/25) a los tres meses $(17,84 / 25)$. Este dato sumado a que disminuye la volatilidad de las desviaciones típicas supone una gran estabilidad de los puntajes obtenidos por los participantes que han cumplimentado el cuestionario (aunque sólo la mitad de los que lo hicieron en el pre-test). Tendríamos que comprobar si sólo los que continúan con las prácticas cotidianas formales e informales son aquellos que han cumplimentado el cuestionario a los tres meses de la finalización del curso o a pesar de la contestación de seis personas sería representativo del conjunto.

Una de las limitaciones de este estudio es el no tener el contraste de un grupo de control, lo que conlleva que se aconsejen investigaciones complementarias que lo repliquen.

La faceta de la observación es una de las más relevantes para discriminar a los meditadores [35], encontramos investigaciones, que discriminan con el FFMQ que los meditadores frente a los no meditadores tienen mentes más adaptativas y obtienen puntajes más altos en la observación de dichos pensamientos y emociones sin enjuiciar y sin reaccionar [36]. En esta misma línea hay autores, que destacan la mayor capacidad de observación y descripción de la experiencia de los meditadores [20].

\section{Conclusiones}

El entrenamiento de la atención plena, a través del Programa de intervención MBCT de ocho semanas consiguió un impacto positivo en todas y cada una de las facetas mindfulness que mide el cuestionario FFMQ-E, constatando el crecimiento del bienestar psicológico percibido en un grupo de población no clínica cuyos efectos permanecen estables al menos tres meses después de su finalización.

En este estudio podemos apreciar una mejora en la capacidad de observación y ser consciente de todo lo que rodea a la persona momento a momento. También se acrecienta la competencia de explicar cómo uno se siente en un momento dado. Aumenta la percepción de la actuación consciente, que cuenta los momentos en los que se presta atención a lo que se está haciendo y no se lleva a cabo de manera automática. Progresa el no enjuiciamiento de las emociones, experiencias o pensamientos, sean agradables o desagradables, manteniendo la ecuanimidad de una manera amable y comprensiva. Se incrementa la no reactividad a la experiencia, haciendo posible una respuesta consciente. 
Agradecimientos: Decanato de la Facultad de Medicina y Ciencias de la Salud de la Universidad de Alcalá.

Conflictos de Intereses: El autor fue director del curso que sirvió de base al estudio.

Consideraciones éticas: Al no estar realizando un ensayo clínico con medicamento, los resultados que se exponen en el test de validación FFMQ, no están sujetos a la normativa; Reglamento (UE) № 536/2014 Parlamento Europeo y del Consejo de 16 de abril de 2014 sobre los ensayos clínicos de medicamentos de uso humano, y por el que se deroga la Directiva 2001/20/CE y validada con el Real Decreto Legislativo 1/2015, de 24 de julio, por el que se aprueba el texto refundido de la Ley de garantías y uso racional de los medicamentos y productos sanitarios.

\section{Abreviaturas}

Las siguientes abreviaturas son usadas en este manuscrito:

FFMQ: Cuestionario mindfulness de las cinco facetas.

MBCT: Mindfulness basado en terapia cognitiva.

MBI: Intervención basada en mindfulness.

MBI-TAC: Criterios de evaluación de enseñanza de la intervención basada en mindfulness.

MBSR: Mindfulness basado en la reducción del estrés.

REBAP: Reducción del estrés basado en la atención plena.

UAH: Universidad de Alcalá.

\section{Referencias Bibliográficas}

1. Kabat-Zinn J. Vivir con plenitud las crisis. Cómo utilizar la sabiduría del cuerpo y la mente para enfrentarnos al estrés, el dolor y la enfermedad. Barcelona: Kairós, 2016.

2. Campos D, y Cebolla A. Enseñar Mindfulness: Contextos de Instrucción y Pedagogía. Revista de Psicoterapia, 2016; 27(103): 103-118.

3. Miró MT, y Simón V. Mindfulness en la práctica clínica. Bilbao: Desclée de Brouwer, 2012.

4. Siegel D. Cerebro y mindfulness: La reflexión y la atención plena para cultivar el bienestar. Ed. Transiciones, 2010.

5. Luis-Pascual JC. Escuela relajada, mindfulness y educación. En JC Bizelli y CB Gomide de Souza (Org.). Faces da escola em Ibero-América. Araraquara, Brasil: Laboratorio editorial da FCL, 2014, 165-180.

6. Ferrer J. Mindfulness, empresas y organizaciones. Terapia de choque: Barcelona, 2016.

7. Mañas I, Aguilas J, Franco C, Gil MD, y Gil D. Mindfulness y rendimiento deportivo. Psychology, Society \& Education, 2014; 6(1): 41-53.

8. Segal ZV, Williams MG, y Teasdale JD. MBCT. Terapia cognitiva basada en el mindfulness para la depresión. Barcelona: Kairós, 2017.

9. Hölzel BK, Lazar, SW, Gard T, Schuman-Olivier Z, Vago DR, y Ott U. How does mindfulness work? Proposing mechanisms of action from a conceptual and neural perspective. Perspectives and Psychological Science, 2011; 6(6): 537-559.

10. Keng SL, Smoski MJ, y Robins CJ. Effects of mindfulness on psychological health: a review of empirical studies. Clinical Psychology Review, 2011;31(6): 1041-56. doi:10.1016/j.cpr.2011.04.006 https://positivepsychologyprogram.com/mindfulness-exercises-techniques-activities/

11. Quintana B. Evaluación del Mindfulness: aplicación del cuestionario Mindfulness de cinco facetas (FFMQ) en población española. (Tesis doctoral). Universidad Complutense de Madrid, España, 2016.

12. Miró-Barrachina MT. La atención plena (Mindfulness) como intervención clínica para aliviar el sufrimiento y mejorar la convivencia. Revista de Psicoterapia, 2006; 17(66-67): 31-76.

13. Krasner MS, Epstein RM, Beckman H, Suchma, AL, Chapman B, Mooney CJ, y Kill TE. Association of an educational program in mindful communication with burnout, empathy, and 
attitudes and primary care physicians. JAMA: Journal of the American Medical Association, $2009 ; 302$.

14. Crane RS, Kuyken W, Mark J, Williams G, Hastings RP, Cooper R, y Fennell MJ. Competence in Teaching Mindfulness-Based Courses: Concepts, Development and Assessment. Mindfulness, 2012; 3: 76-84.

15. García-Campayo J, y Demarzo M. Manual Práctico de Mindfulness. Curiosidad y aceptación. Barcelona: Siglantana, 2015.

16. Delgado-Pastor LC. Correlatos psicofisiológicos de mindfulness y la preocupación. Eficacia de un entrenamiento en habilidades mindfulness. Granada: Universidad de Granada, 2009.

17. Baer RA, Smith G, y Allen K. Assessment of mindfulness by self-report: The Kentucky Inventory of Mindfulness Skills. Assessment, 2004; 11(3): 191-206.

18. Baer RA, Smith GT, Hopkins J, Krietemeyer J, y Toney L. Using self- report assessment methods to explore facets of mindfulness. Assessment, 2006; 13: 27-35.

19. Ruijgrok-Lupton PV, Crane RS, y Dorjee D. Impact of Mindfulness-Based Teacher Training on MBSR Participant Well-Being Outcomes and Course Satisfaction. Mindfulness, 2018; 9: 117-128.

20. Schmidt C, y Vinet EV. Atención Plena: Validación del Five Facet Mindfulness Questionnaire (FFMQ) en estudiantes universitarios chilenos. Terapia psicológica, 2015; 33(2), 93102. https://dx.doi.org/10.4067/S0718-48082015000200004

21. Strege MV, Swain D, Bochicchio L, Valdespino A, y Richey, JA. A Pilot Study of the Effects of Mindfulness-Based Cognitive Therapy on Possitive Affect and Social Anxiety Symptoms. Frontiers in Psychology, 2018; 866: 1-11.

22. Soler J, Tejedor R, Feliu-Soler A, Pascual JC, Cebolla A, Soriano J, Álvarez E, y Pérez V. Propiedades psicométricas de la versión española de la escala Mindful Attention Awareness Scale (MAAS). Actas Esp Psiquiatr, 2012; (40)1: 19-26.

23. Buchheld N, Grossman P, Walach H. Measuring Mindfulness in Insight Meditation and Meditation-Based Psychotherapy: The development of the Freiburg Mindfulness Inventory (FMI). Journal for Meditation and Meditation Research, 2002; 1: 11-34.

24. Walach H, Buchheld N, Buttenmüller V, Kleinknecht N, y Schmidt S. Measuring mindfulnessThe Freiburg Mindfulness Inventory (FMI). Personality and Individual Differences, 2006; 40: 1543-1555.

25. Feldman G, Hayes A, Kumar S, Greeson J, y Laurenceau JP. Mindfulness and Emotion Regulation: The Development and Initial Validation of the Cognitive and Affective Mindfulness Scale-Revised (CAMS-R). J Psychopathol Behav Assess, 2007; 29: 177-190.

26. Chadwick P, Hember M, Mead S, Lilley B, y Dagnan D. Responding mindfully to unpleasant thoughts and images: Reliability and validity of the Mindfulness Questionnaire. Br J Clin Psychol, 2010; 47(4): 451-455.

27. Cardaciotto LA, Herbert JD, Forman EM, Moitra E, y Farrow V. The Assessment of PresentMoment Awareness and Acceptance: The Philadelphia Mindfulness Scale. Assessment, 2008; 15: 204.-223.

28. Lau MA, Bishop SR, Segal ZV, Buis T, Anderson ND, Carlson L, et al. The Toronto Mindfulness Scale: Development and Validation. Journal of Clinical Psychology, 2006; 62: 1445-1467.

29. Cebolla A, García-Palacios A, Soler J, Guillen V, Baños R, y Botella C. Psychometric properties of Spanish validation of the Five Facets of Mindfulness Questionnaire (FFMQ). Eur. J. Psychiat, 2012; 26(2): 118-126. http://dx.doi.org/10.4321/S0213-61632012000200005.

30. Martínez-Lentisco MM, Espinoza-Serrano RL, y Sola-Sola E. Aprendiendo a parar. El mindfulness como herramienta de bienestar. International Journal of Developmental and Educational Psychology, INFAD Revista de Psicología, 2017; 1, Monográfico 3: 215-222.

31. Hyland T. McMindfulness in the workplace; vocational learning and the commodification of the Present Moment. Journal of Vocational Education \& Training, 2015; 67(2): 219-234.

32. Zeidan F, Martucci KT, Kraft RA, Gordon NS, McHaffie JG, y Coghill RC. Brain Mechanisms Supportin the Modulation of Pain by Mindfulness Meditation, The Journal of Neuroscience, 2011; 31(4): 5540-5548. 
33. Luis-Pascual JC. Tendencias y controversias del mindfulness educativo en España. En M MartínBris, MC Miránda-Álvarez, E Sebastián Heredero, y R Edel-Navarro (Coords.). Visiones de la educación en Iberoamerica: Experiencias y reflexiones. Tomo II: Tópicos emergentes de la educación. Veracruz, México: Universidad Veracruzana, 2016: 46-61.

34. Chiesa A, y Serretti A. Mindfulness-based stress reduction for stress management in healthy people: a review and meta-analysis. Journal of Alternative and Complementary Medicine, 2009; 15(5): 593-600. doi:10.1089/acm.2008.0495.

35. Lilja JL, Lundh L, Josefsson T, y Falkenström F. Observing as an essential facet of mindfulness: A comparison of FFMQ patterns in meditating and non-meditating individuals. Mindfulness, 2013; 4(3): 203-212.

36. Lykins EL, y Baer R. Psychological Functioning in a Sample of Long-Term Practitioners of Mindfulness Meditation. Journal of Cognitive Psychotherapy, 2009; 23(3): 226-241. doi:10.1891/0889-8391.23.3.226

(C) 2019 por los autores; Esta obra está sujeta a la licencia de Reconocimiento 4.0

Internacional de Creative Commons. Para ver una copia de esta licencia, visite http://creativecommons.org/licenses/by-nc-nd/4.0/. 